\title{
Self-Organized InGaAs Quantum Rings - Fabrication and Spectroscopy -
}

Axel Lorke ${ }^{1}$, Jorge M. Garcia ${ }^{2}$, Ralf Blossey ${ }^{3}$, Richard J. Luyken ${ }^{4}$, and Pierre M. Petroff ${ }^{5}$

1 Experimentalphysik, Universität Duisburg-Essen, Lotharstr. 1 ME245, 47048 Duisburg, Germany

2 Instituto de Microelectrónica, Parque Tecnologico de Madrid, 28760 Tres Cantos, Madrid, Spain

3 Interdisciplinary Research Institute, IEMN, Cité Scientifique Avenue Poincaré, BP 69, 59652 Villeneuve d'Ascq Cedex, France

${ }^{4}$ Infineon Technologies, Corporate Research ST, Otto Hahn Ring 6, 81739 München, Germany

${ }^{5}$ Materials Department, University of California, Santa Barbara Ca. 93106, USA

\begin{abstract}
We report on recent experiments on semiconductor quantum rings, fabricated by self-organized Stranski-Krastanov growth. In the first part of this review we focus on the growth mechanisms that lead to formation of annular islands, with emphasis on the influence of diffusion and surface tension. In the second part, we summarize the results of intraband spectroscopy, which show the influence that the ring morphology has on the electronic properties, in particular, when a magnetic flux quantum threads the interior of the islands.
\end{abstract}

\section{Introduction}

When Goldstein et al. [1] in 1985 reported on the observation of island formation in the Stranski-Krastanov growth of InAs on GaAs, their work was more concerned with the optimization of smooth (InGa)As layers for optically active quantum wells than with the fabrication of nanostructures. They did however already observe all ingredients of what was to become a great breakthrough for semiconductor nanoscience: bright luminescence out of islands of just the right size for single-electron and quantum effects.

Soon the benefits of these (InGa)As islands as self-ordering semiconductor nanostructures were realized and the optical, far-infrared and transport properties were studied in great detail. It was shown that the ease of fabrication, the homogeneity and the crystal quality made self-organized InAs islands ideal systems to study many-particle quantum effects in electronic and excitonic systems with a precision that the label "artificial atoms" was indeed warranted for these semiconductor nanostructures [2].

The terms "self-organized" or "self-assembled" seem to imply that within the parameter-space dictated by the optimum growth conditions, the morphology of the islands is more or less fixed. This would certainly limit the 
use of these islands as model systems for quantum effects on the nanometer scale. It was soon realized though, that additional ordering mechanisms can be exploited for more complicated shapes. The most prominent among these mechanisms is the vertical ordering that can lead to double (or multiple) vertically aligned dots [1,3,4], often labeled as "artificial molecules". In the strained Si-Ge-system, Stranski-Krastanov growth can even lead to three-dimensionally ordered lattices [5].

A further method to vary the shape of (InGa)As islands makes use of a morphological change that takes place when the dots are partially covered with GaAs. As will be discussed in detail below, then islands with a welldeveloped ring-shape can be fabricated.

Rings are distinctly different from single or multiple stacked dots in that they have a not-simply-connected geometry. This geometry makes them particularly interesting for studies in magnetic fields. The quantization of magnetic flux in the interior leads to a periodic change in the quantum mechanical properties of the electron system encircling the ring. This in turn affects all electronic properties of rings. For sufficiently narrow rings, these oscillations are periodic in the applied magnetic field and are commonly labeled "Aharonov-Bohm-oscillations", even though - strictly speaking- the Aharonov-Bohm effect requires the absence of a magnetic field along the path of the electrons. In 1959 Aharonov and Bohm discussed [6] that even in this case, the electron acquires an additional phase along its path that is directly proportional to the vector potential $\boldsymbol{A}$. For a closed loop, where the total phase must be an integer multiple $n$ of $2 \pi$, the discreteness of $n$ even when $\boldsymbol{A}$ is changed continuously leads to oscillations that have no classical analogue.

The theoretical predictions were soon experimentally confirmed and Aharonov-Bohm-oscillations have since been observed in a large variety of systems, mostly, though in homogeneous magnetic fields fields where $\boldsymbol{B} \neq 0$ along the path of the electrons [7-9]. Also, most of the experiments have been carried out in the semiclassical regime where the electron wavelength is given by the Fermi-energy of the chosen material. Furthermore, most experiments were carried out in the mesoscopic regime, where (non-phase-breaking) scattering plays an important role in the determination of the electron path.

Naturally occurring ring-like quantum structures like the benzene molecule seem to offer an alternative to the above-mentioned semi-classical systems. In order to thread a flux-quantum through the interior of a benzene molecule, however, magnetic fields of around $50000 \mathrm{~T}$ are needed, orders of magnitude more than the fields available in today's laboratories.

Self-organized quantum rings have all properties necessary to investigate flux-related phenomena in fully quantized, scatter-free few-electron systems. Their lateral size is large enough so that they can encompass a flux quantum in magnetic fields of a few tesla. Also, they can be controllably charged with single electrons, so that the influence of the electron-electron-interaction on the Aharonov-Bohm-oscillations can be studied. Furthermore, their small- 
ness, their superb crystal quality and the fact that they can be probed as an ensemble average ensures that random scattering effects do not play a decisive role in the observed features. Finally, self-organized quantum rings are experimentally accessible through a number of techniques. The fact that transport, far-infrared and optical interband spectroscopy can be performed on the same sample allows for an in-depth characterization of the electronic and excitonic structure. This way, a complete picture, containing the influence of the external confining potential, the electron-electron-interaction and the magnetic field on the many-particle states can be derived.

In this review we will first focus on the formation of self-organized (InGa)As quantum rings. After summarizing the growth conditions necessary for ring formation, we will discuss the implications that the transformation from dots to rings has on the understanding of Stranski-Krastanov growth in III-Vsemiconductors. In the second part, we will review the electronic properties of ring-shaped quantum structures in magnetic fields and show how these are related to the experimental observations in transport and far-infrared spectroscopy.

\section{Growth Procedure}

In hindsight, the observation of well-defined ring-shaped islands was to some extend a fortunate coincidence. We now know that a number of growth parameters have to be chosen correctly to induce the ring formation. The rings shown in Fig. 1 were prepared in the following way [10] (see also Fig. 2).

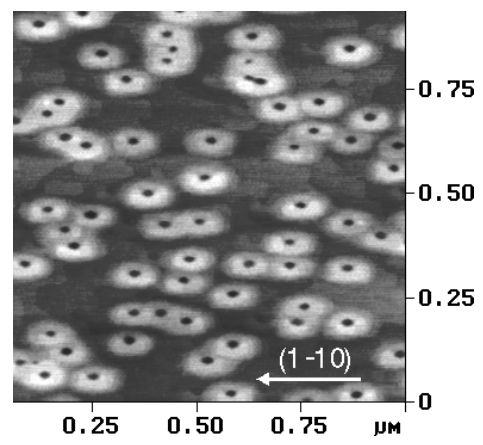

Fig. 1. Atomic-force micrograph of self-organized (InGa)As quantum rings. Note the slight elongation along the (110)-direction

On silicon-doped GaAs (100) wafers, a GaAs buffer layer was grown at $\approx 600^{\circ} \mathrm{C}$ to smooth out the substrate. Then the temperature was lowered to $\approx 530^{\circ} \mathrm{C}$, a value that can be reproduced in different growth chambers by observing the RHEED pattern, since under moderate As flux, at this 
(a)

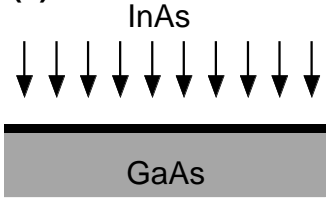

$530{ }^{\circ} \mathrm{C}$

(c)

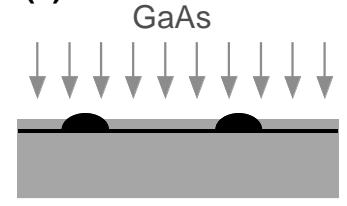

GaAs cover layer (b)

$\approx 1.7$ monolayers

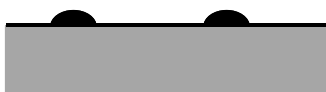

dots

(d)

$\approx 30$ s anneal

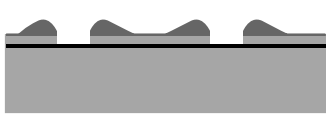

rings

Fig. 2. Growth sequence used for the fabrication of (InGa)As rings

temperature the surface reconstruction changes from $(2 \times 4)$ to $c(4 \times 4)$. Then, 1.7 monolayers of InAs were deposited at a low growth rate to induce Stranski-Krastanov formation of large, approximately lens-shaped islands. In the present case, the islands have a base width of approximately $30 \mathrm{~nm}$ and a height of about $10 \mathrm{~nm}$. Our studies [11] and those of other authors [12-14] strongly suggest that large dots are a necessary starting condition for the formation of well-defined rings. The sample was annealed for $40 \mathrm{~s}$ under As flux to narrow the size distribution and further help the formation of large islands [16]. The dots were then capped with $4 \mathrm{~nm}$ GaAs and the wafers were -after an annealing time of a few 10 seconds- cooled down to room temperature under As flux and removed from the chamber for AFM characterization under ambient conditions.

As seen in Fig. 1, this procedure results in well-defined rings of $\approx 2 \mathrm{~nm}$ height, $20 \mathrm{~nm}$ inner diameter and between 60 and $140 \mathrm{~nm}$ outer diameter. Detailed statistical analysis [17] shows that the centers of the rings are located at the sites of the former dots. Even though this does not seem very surprising, it shows that the annular mounds are formed by an outward transport of material that leads to a dissolution of the original, lens-shaped island. In the following section we will more closely analyze the different mechanisms that are responsible for this outward movement of the In-rich material. 


\section{Growth Mechanisms}

One possible reason for the redistribution of the InAs is diffusion. At the growth temperature of $\approx 530^{\circ} \mathrm{C}$, the In atoms are very mobile on the surface [18], whereas the Ga atoms can diffuse only little during the growth interruption. Both is reflected in the shape of the rings. The outer boundary is elongated along the (110)-crystal orientation, which is the preferred direction for In-diffusion on the reconstructed GaAs (100) surface [18]. The inner boundary, on the other hand, remains circular, which shows that the GaAs that is surrounding the dots just after the capping layer deposition (see Fig. 2(c)), remains in place (Fig. 2(d)). The elongation of the outer edge and the almost circular inner edge can even better be seen in capped InAs islands, grown under somewhat different growth conditions [19-21].
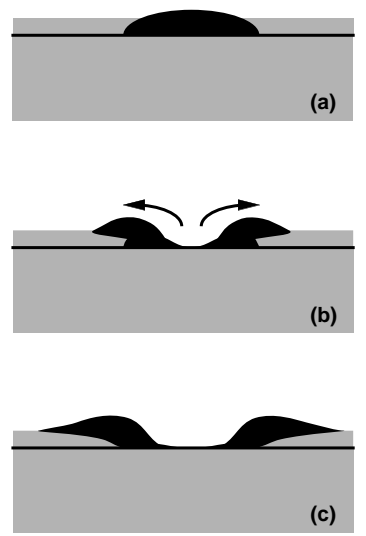

Fig. 3. Model of diffusion-driven ring formation. See text for details. After Ref. [23]

A possible scenario for the ring formation is depicted in Fig. 3 [21]. Here, -as in Fig. 2- it has been assumed that the GaAs capping layer is surrounding the dot rather than covering it like a blanket (see Fig. 3(a)). This assumption is commonly made in the literature and reflects the fact that the relaxed InAs lattice constant at the tip of the dot [22] makes it an unfavorable site for the attachment of $\mathrm{Ga}$ atoms. It is further confirmed by transport measurements [23] which show that only when the capping layer thickness is comparable to the height of the original dot, the In is locked in place by the GaAs coverage and the annealing step no longer results in ring formation. A further assumption made in Fig. 3 may on the other hand not be justified. In step (b) and (c) the outdiffusing In stays more or less fixed once it has left the site of the dot. Only then the relatively sharp outer edge of the rings can be obtained. Even though this might be explained by (InGa)As alloy formation [23], we believe that diffusion alone cannot account for all experimental evidence. 
Apart from the sharp ring edge mentioned above, we observe that the ring formation seems to take place quite abruptly [21] rather than gradually, as expected for a diffusive mechanism. The strongest evidence against a picture that is based on different diffusion constants of the group III constituents comes from the work of Raz et al. [14]. They have shown that self-assembled In As rings can also be grown on InP. In this material combination, the substrate and the capping layer contain the same group III element as the dots. Starting from Fig. 3(a) and only assuming In migration, a depression in the center as shown in Fig. 3(b) or (c) cannot be explained.

An alternative explanation for the abrupt morphological change of the InAs islands comes from an analogy to the instability of wetting droplets. In seemingly unrelated material combinations (e.g. polystyrene on Si), structures of striking similarity to the present rings have been observed and explained by a dewetting process [24]. It should also be remembered that one of the key ingredients for the understanding of Stranski-Krastanov growth is the interplay between surface and interface energies of the different constituents. The same should be true for partially capped islands.

(a)

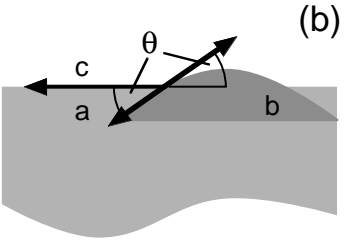

(c)

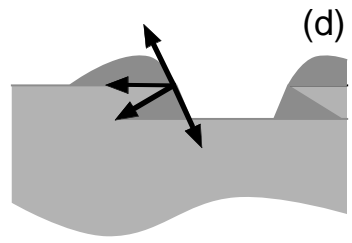

Fig. 4. Model for ring formation based on wetting droplet instability. After Ref. [25]

An explanation for (InGa)As ring formation, based on dewetting phenomena, is given in Fig. 4 [25]. As shown in (a), the wetting angle $\theta$ of uncapped islands is given by the balance of lateral forces at the base of the dot,

$$
\gamma_{a c}=\gamma_{b c} \cos (\theta)+\gamma_{a b} .
$$


Here, $\gamma_{i j}$ is the force at the interface between materials $i$ and $j$, which in the present case are $a=\mathrm{GaAs}, b=\mathrm{InAs}$, and $c=$ As-vapor. When the islands are partially capped, the corresponding relation would read (see Fig. 4(b))

$$
\gamma_{a c}=\gamma_{b c} \cos (\theta)-\gamma_{a b} \cos (\theta) \text {. }
$$

Since eq. (2) is incompatible with eq. (1), the configuration shown in Fig. 4(b) cannot be in equilibrium and a net force $\Delta \boldsymbol{F}$ must be present for partially capped islands (Fig. 4(c)). This radial force can explain the outward motion of InAs that leads to the ring formation. A possible new equilibrium shape of the rings is suggested in Fig. 4(d). Here, the (lateral) interfacial forces are balanced again and the shape is in qualitative agreement with the experimental data.

In this model, we have for simplicity neglected the presence of the InAs wetting layer that is connecting the dots (see Fig. 2). The wetting layer will certainly affect the details of the ring formation. However, it will not qualitatively change the results [25]. Still, it would be desirable to develop a more sophisticated model that would also include the wetting layer as well as strain. Strain is known to play an important role in determining the equilibrium shape of (InGa)As dots. First steps towards a more complete model are being taken $[13,15]$.

\section{Further Control of the Island Shape}

As discussed in the previous section, a number of effects play a role in the formation of self-organized quantum rings, among them diffusion, strain, and surface and interface energies. All of these can be controlled by proper choice of the growth parameters. This way, not only rings but other complex shapes can be realized.

The influence of diffusion on the shape of capped InAs islands was investigated in Ref. [11]. Diffusion can be influenced not only by changing the growth temperature but also by using cracked $\mathrm{As}_{2}$, which is more reactive than $\mathrm{As}_{4}$ and thus reduces the mobility of the group III elements. This way, structures ranging from elongated islands to rings could be realized. An intermediate structure, i.e. an elongated island with a depression in its center is of particular interest. Such a "camel back" structure might be useful for realizing double dot systems with molecule-type electronic properties.

Strain and interface energies can be influenced by covering the dots not with pure GaAs but with $\operatorname{In}_{x} \mathrm{Ga}_{1-x}$ As alloy of different composition $x$. As shown by Songmuang et al. [13], then the dissolution of the center dot can be completely suppressed and structures of surprising complexity emerge, such as a center island with a surrounding mound and a thin trench separating the two.

Even though this demonstrates that an impressive variety of structures can be realized using self-organized growth, an important question remains. 
If indeed the overgrowth plays such an important role in the distribution of material, can the shape of the freestanding structures be preserved during the next growth steps that are necessary for the fabrication of electrically and optically active structures?

In the next section we will show that this is true, at least for ( $\mathrm{InGa}$ ) As rings, by demonstrating that the electronic properties of the completed heterostructure are reflecting the ring geometry of the islands.

\section{$5 \quad$ Electronic Properties}

To study their electronic properties, the (InGa)As rings are embedded into a capacitor-type MISFET structure (metal-insulator-semiconductor field-effect transistor structure). The basic layer sequence is schematically depicted in Fig. 5 .

First, a heavily Si-doped back contact layer is grown, which serves as an electron reservoir and defines the local Fermi level. It is followed by a $25 \mathrm{~nm}$ GaAs spacer that separates the islands from the doping layer but allows for tunneling of electrons between the back contact and the rings. The rings themselves are grown using the above described Stranski-Krastanov procedure, with a capping layer thickness of $1 \mathrm{~nm}$. They are then covered with $30 \mathrm{~nm}$ GaAs, followed by a $116 \mathrm{~nm}$ blocking layer that prevents tunneling or leakage currents between the rings and the top gate electrode. The blocking layer consists of a AlAs/GaAs superlattice, the gate is realized by a semi-transparent $\mathrm{NiCr}$ layer. The total area of the sample is about $5 \mathrm{~mm}^{2}$, covering $\approx 5 \times 10^{8}$ rings. The low density ensures that the rings are well separated laterally and that to a good approximation the charge in the ring layer can be neglected. Then an external voltage (shifted by the built-in voltage caused by the Schottky barrier) will cause a linear electrostatic potential across the structure, as schematically depicted in Fig. 5(bottom). This way, the applied gate voltage $V_{g}$ (scaled by the "lever arm" $t_{\text {tot }} / t_{1}$, see Fig. 5) directly translates into an energy shift of the rings with respect to the back contact. Single electrons are loaded into the rings, each time the energy of the corresponding state is shifted below the Fermi energy in the back contact. This can be probed by application of an additional AC voltage $V_{\text {mod }}$. On resonance, electrons are tunneling back and forth between the rings and the back contact, leading to an effectively increased capacitance of the structure. Alternatively, the increase in the capacitance can be understood as the contribution of the so-called quantum capacitance [26], which is proportional to the electronic density of states in the rings. Thus, the capacitance-voltage (CV) traces directly reflect the many-particle ground state energies of the quantized electronic system.

Complementary information can be obtained by far-infrared transmission spectroscopy, which probes the excitations of the confined electron system. Due to the extremely low total number of electrons in the rings, the absorp- 

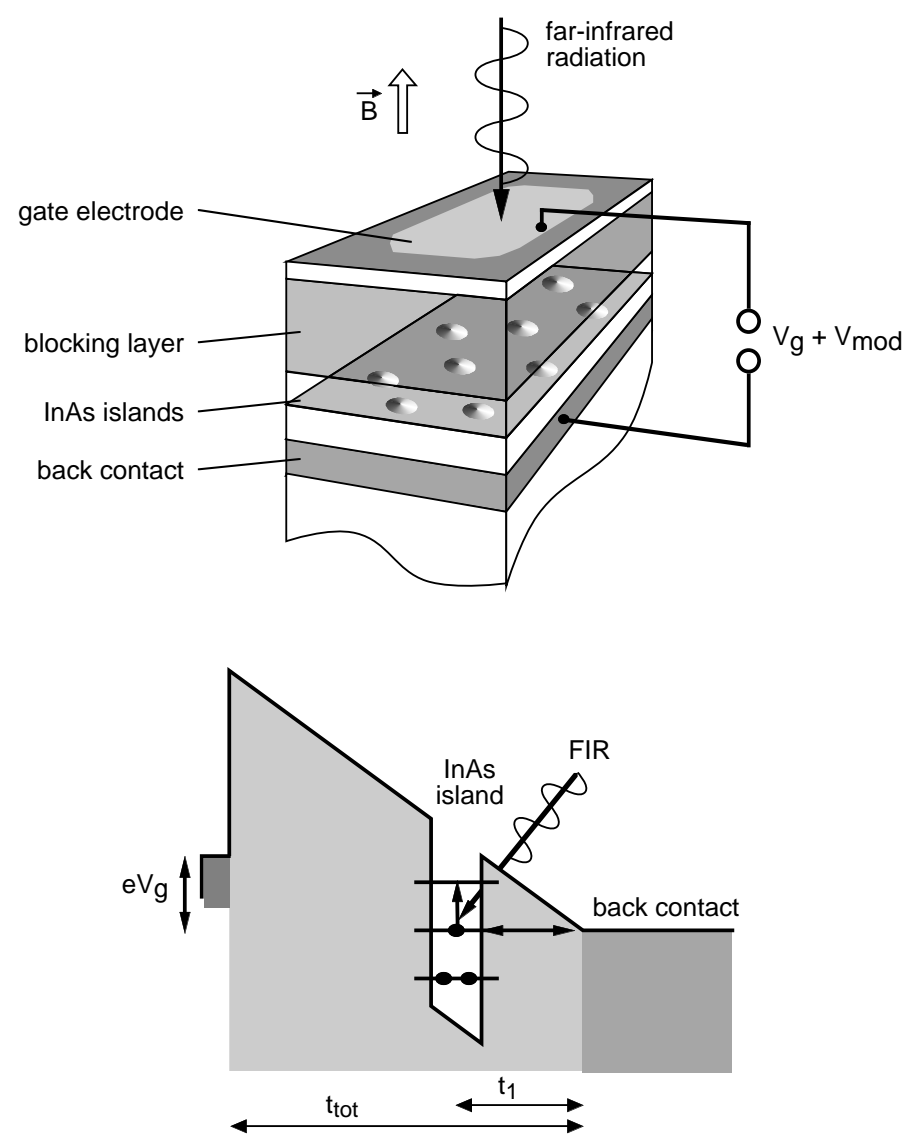

Fig. 5. (Top) Schematic layer structure and experimental geometry used for the spectroscopic experiments. (Bottom) Simplified sketch of the conduction band structure

tion is of the order of $0.1 \%$, which constitutes a great experimental challenge in the far-infrared. The tunability of the electron number is of great advantage here, because signal-to-noise ratios down to $10^{-4}$ can be achieved when the sample spectrum is normalized to a spectrum where the gate voltage is chosen so that the rings are void of electrons.

Figure 6 shows a $\mathrm{CV}$-spectrum of the above described quantum ring heterostructure. Around $V_{g}=0 \mathrm{~V}$, two maxima (arrows) indicate the loading of the first and second electron per dot. At higher voltages, the capacitance rapidly increases because of the loading of the wetting layer [27] and no further charging peaks can be discerned in this sample (on other samples, however, a third maximum can be seen [28]). In comparison to the spectrum of dots [29] (see inset), the first charging peak of rings is shifted to 


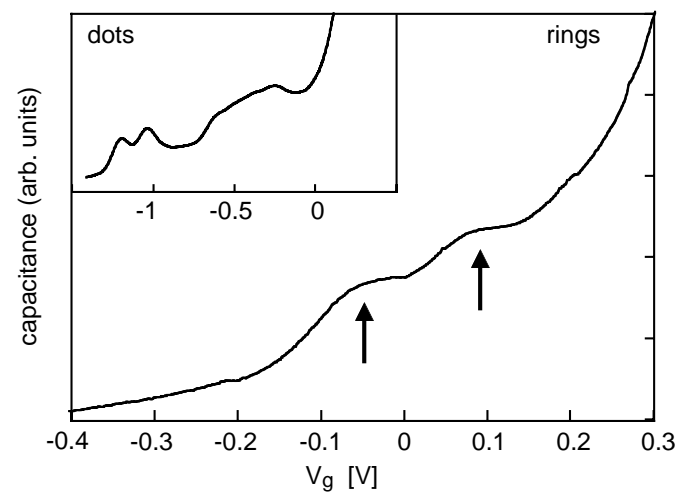

Fig. 6. Capacitance-voltage traces of self-organized rings and dots. Arrows indicate the charging of the first and the second electron per ring

higher voltages. This reflects the reduced height of the rings, which increases the electronic ground state energy. The lowest state is doubly spin degenerate and the separation between the first and the second maximum is given by the electron-electron-interaction. Concerning the charging energy, the difference between dots and rings is surprisingly small, considering the large difference in lateral size (about a factor of three). This might already be an indication of the missing center part of the rings, which would decrease the effective area available for the electrons and thus increase their interaction strength. It should be pointed out, though, that the conversion of voltages into energies is not very reliable in the ring sample, because the lever arm (nominally 7 in the present case) is not well defined close to the charging of the wetting layer.

As mentioned in the introduction, a direct way to show that the notsimply-connected geometry of the islands translates into ring-like electronic properties is the investigation of the magnetic properties of the sample. Figure 7 (a) summarizes the measured far-infrared resonances as a function of an external magnetic field $B$, applied perpendicular to the plane of the rings [30]. The resonances can be grouped into the following modes: (1) two resonances (0) which are degenerate at $B=0$ and exhibit orbital Zeeman splitting when the magnetic field is applied; (2) a low-lying mode $(\diamond)$ which -because of the limitations of the spectrometer- can only be detected above $10 \mathrm{meV}$, but extrapolates to $\approx 7 \mathrm{meV}$ at $B=0$. Both the $(\diamond)$ - and the $(0)$-mode die out around $B=7 \mathrm{~T}$, when a new mode $(\triangle)$ appears.

The appearance and disappearance of modes is in contrast to the properties of quantum dots [2], where in general, only two resonances are observed, 


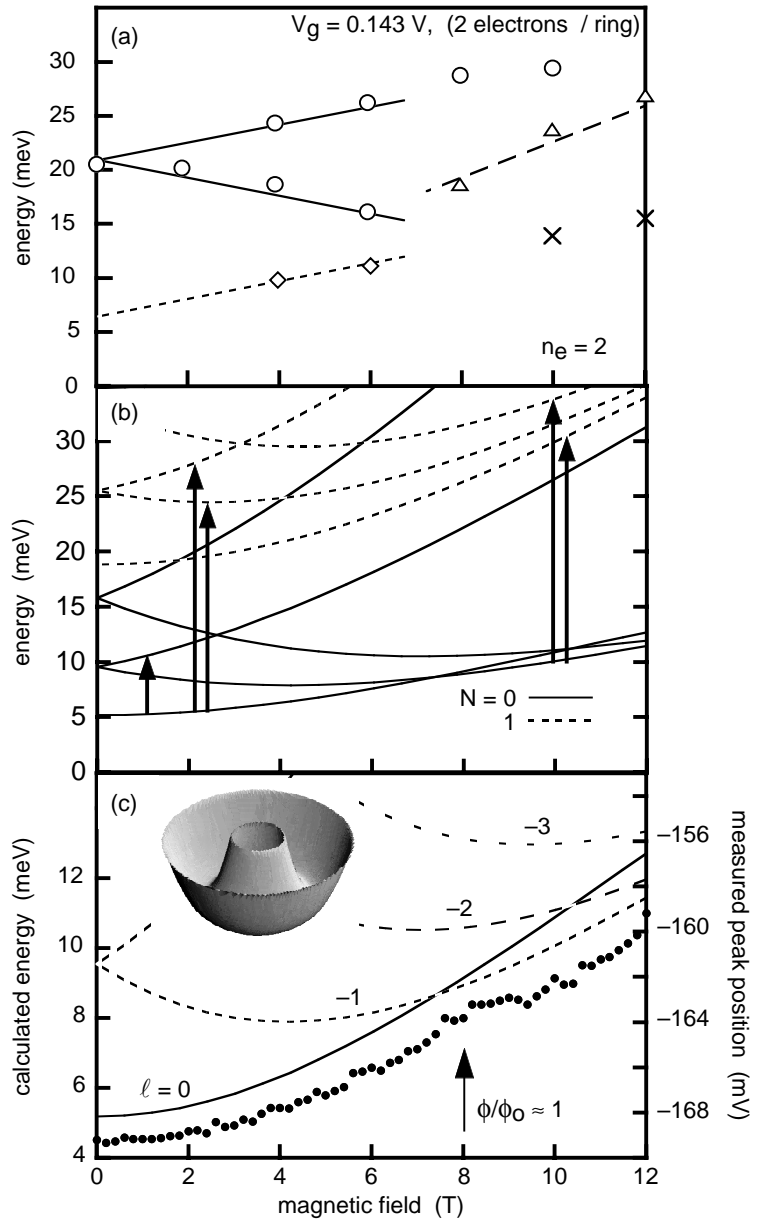

Fig. 7. (a) Far-infrared resonance energies of quantum rings. Lines represent linear Zeeman terms corresponding to half the cyclotron energy (solid, dotted lines) and the full cyclotron energy (dashed line), respectively, see Ref. [30]. The mode indicated by $\times$ cannot be accounted for and might be an artifact. (b) Possible excitations from the ground state, assuming selection rules $\Delta N=0,1, \Delta \ell= \pm 1$. Note the change in ground state that occurs just below $8 \mathrm{~T}$. (c) Lines: Calculated ground state energies of quantum rings as a function of the magnetic field. The insert displays the model potential used for the calculations. Dots: Experimental values of the lowest $\mathrm{CV}$ charging peak 
which are separated by the Zeeman energy. The periodic exchange of the allowed resonances in a magnetic field is, on the other hand, a characteristic of (sufficiently narrow) quantum rings.

Making use of a model by Chakraborty et al. [31], we calculate the single electron states in a confining potential

$$
U(r)=\frac{1}{2} m^{*} \omega_{0}^{2}\left(r-R_{0}\right)^{2},
$$

where $\omega_{0}$ is the characteristic frequency of a parabolic wire, bent into a ring of radius $R_{0}$ (see inset in Fig. $7(\mathrm{c})$ ). This model has the advantage that it requires only two adjustable parameters, which can readily be fitted to the (extrapolated) resonances at $B=0$, one of which $(\approx 20 \mathrm{meV})$ is given the radial confinement, whereas the other $(\approx 7$ meV) reflects an azimuthal excitation with a change in angular momentum. The derived parameters, $R_{0}=14 \mathrm{~nm}, \hbar \omega_{0}=12 \mathrm{meV}$ can be used to calculate the energy levels for the entire range of magnetic fields investigated. The result is shown in Fig. 7(b), where the energies of the states with radial quantum number $n=0$ and angular momentum $\ell=0, \pm 1, \pm 2$ (solid lines) as well as with $n=0, \ell=$ $0, \pm 1,-2$ (dashed lines) are plotted. The arrows in Fig. $7(\mathrm{~b})$ indicate possible far-infrared excitations from the lowest energy state, under the assumption that only transition with $\Delta N=0,1$ and $\Delta \ell= \pm 1$ are allowed.

Even though the fit parameters were obtained from the resonance positions at $B=0$, the calculated dispersion is in qualitative agreement with the experiment also at high magnetic fields. Here, the most important feature is the predicted change in ground state at a magnetic field of around $7 \mathrm{~T}$. This change from a state with zero angular momentum to a state with angular momentum $\ell=-1$ is responsible for the appearance and disappearance of the different resonances shown in Fig. 7(a).

The strongest evidence for a change in angular momentum at high magnetic fields comes from a direct mapping of the single-electron ground state energy by capacitance spectroscopy. Figure Fig. 7(c) shows the results of a careful fitting procedure to accurately determine the lowest charging peak position. Up to approximately $8 \mathrm{~T}$, the scaled data closely follow the parabolic dispersion of the $\ell=0$ state. At magnetic fields above $8 \mathrm{~T}$ the slope of the magnetic field dependence changes and now the data is in good agreement with the dispersion of the $\ell=-1$ state.

Summarizing the results shown in Fig. 7, three independent experimental observations can simultaneously be described using the model parameters $R_{0}=14 \mathrm{~nm}, \hbar \omega_{0}=12 \mathrm{meV}$ : The resonance positions at $B=0$, the change in the far-infrared excitation spectrum at $\approx 8 \mathrm{~T}$ and the change in slope of the lowest charging peak at about the same field. This gives us confidence in the validity of the model and shows that indeed, the ring morphology of the (InGa)As islands translates into annular electronic states.

So far, we have restricted ourselves to a single-particle picture. A lot of interesting physics, however, arises from the influence of the Coulomb inter- 
action on the ground state and the excitations. For example, two electrons confined to a ring can be understood as a rotating Wigner molecule [32]. At present, the large number of rings that are probed simultaneously does not allow us to complement the theoretical predictions with experimental data of sufficient resolution. Great progress in interband optical spectroscopy, on the other hand, has made it possible to investigate single rings and study the influence of electron-electron interaction with high resolution [33]. These experiments were carried out at $B=0$ and the challenge remains to apply a high magnetic field and observe clear evidence for the ring morphology. Progress in this direction is rapid [34], so that the observation of (somewhat counter-intuitive) Aharonov-Bohm-type phenomena in neutral excitons [35] seems feasible.

Also, the calculated ground state spectra for a many-electron system in a quantum ring display characteristic features, caused by the electron-electron interaction. For two electrons, the ground state should exhibit a periodic change between spin singlet and triplet states [36]. The results of the $\mathrm{CV}$ spectra for 2 electrons (not shown here) are in rough agreement with many particle calculations [37], a clear confirmation of the theoretical predictions, however, is still lacking.

Refinements in both the theoretical and experimental tools promise for the future many unexpected and interesting results that will deepen the understanding of the fascinating objects of nanoscopic rings in the quantum limit.

\section{Acknowledgement}

The work summarized here was accompanied by continuous support and by stimulating discussions with A. O. Govorov, R. J. Warburton, K. Karrai S. E. Ulloa, and J. P. Kotthaus. We would like to thank them for their fruitful collaboration. Financial support through SFB 348 and BMBF grant 01BM164 is gratefully acknowledged.

\section{References}

1. L. Goldstein, F. Glas, J.-Y. Marzin, M. N. Charasse, and G. Le Roux, Appl. Phys. Lett. 47, 1099 (1985).

2. for reviews, see, e.g., S. M. Reimann and M. Manninen, Rev. Mod. Phys. 74, 1283 (2002); D. Bimberg, M. Grundmann, N. N. Ledentsov, Quantum Dot Heterostructures, (John Wiley, Chichester 1999).

3. W. Wu et al., Appl. Phys. Lett. 71, 1083 (1997).

4. R. J. Luyken et al., Nanotechnology 10, 14 (1999).

5. V. Holy et al., Phys. Rev. Lett. 83, 356 (1999).

6. Y. Aharonov and D. Bohm, Phys. Rev. 115, 485 (1959). 
7. L. P. Lévy et al., Phys. Rev. Lett. 64, 2074 (1990); V. Chandrasekhar et al., ibid., 67, 3578 (1991); D. Mailly, C. Chapelier, and M. Benoit, ibid., 70, 2020 (1993); A. F. Morpurgo et al., ibid., 80, 1050 (1998); R. Schuster et al., Nature 385, 417 (1997).

8. A. Fuhrer et al., Nature 413, 822 (2001).

9. U. F. Keyser et al., Semicond. Sci. Tech. 17, L22 (2002).

10. J. M. García et al., Appl. Phys. Lett. 71, 2014 (1997).

11. D. Granados and J. M. García, Appl. Phys. Lett., accepted.

12. P. B. Joyce et al., Appl. Phys. Lett. 79, 3615 (2001).

13. R. Songmuang, S. Kiravittaya, and O. G. Schmidt, J. Cryst. Growth 249, 416 (2003)

14. T. Raz, D. Ritter, and B. Bahir, Appl. Phys. Lett. 82, 1707 (2003).

15. L. G. Wang et al., Appl. Phys. A 73, 161 (2001).

16. F. Ferdos et al., Appl. Phys. Lett. 81, 1195 (2002).

17. K. Mecke, private communication (unpublished).

18. V. Bressler-Hill et al., Phys. Rev. B 50, 8479 (1994) and references therein.

19. I. Kamiya, I. Tanaka, and H. Sakaki, J. Cryst. Growth 201, 1146 (1999).

20. H. Heidemeyer et al., Appl. Phys. Lett. 80, 1544 (2002).

21. A. Lorke et al., Mat. Sci. Eng. B 88, 225 (2002).

22. I. Kegel et al., Phys. Rev. Lett. 85, 1694 (2000).

23. A. Lorke et al., Jpn. J. Appl. Phys. 40, 1857 (2001).

24. S. Herminghaus et al., Science 282, 916 (1998)

K. Jacobs and S. Herminghaus, Physikalische Blätter 55 (12), 35 (1999)

25. R. Blossey and A. Lorke, Phys. Rev. E 65, 021603 (2002).

26. R. J. Luyken et al., Appl. Phys. Lett. 74, 2486 (1999).

27. G. Medeiros-Ribeiro, D. Leonard, and P. M. Petroff, Appl. Phys. Lett. 66, 1767 (1995)

28. H. Pettersson et al, Physica E 6, 510 (2000).

29. see, e.g. M. Fricke et al., Europhys. Lett. 36, 197 (1996); B. T. Miller et al., Phys. Rev. B 56, 6764 (1997) and references therein.

30. A. Lorke et al., Phys. Rev. Lett. 84, 2223 (2000).

31. T. Chakraborty and P. Pietiläinen, Phys. Rev. B 50, 8460 (1994); V. Halonen, P. Pietiläinen, and T. Chakraborty, Europhys. Lett. 33, 377 (1996).

32. L. Wendler et al., Z. Phys. B 100, 211 (1996).

33. R. J. Warburton et al., Nature 405, 926 (2000).

34. D. Haft et al., Physica E 13, 165 (2002).

35. A. O. Govorov et al., Phys. Rev. B 66, 081309(R) (2002).

36. H. Hu, J-L. Zhu, and J-J. Xiong, Phys. Rev. B 62, 16777 (2000).

37. A. Emperador et al., Phys. Rev. B 62, 4573 (2000). 